EPJ Web of Conferences 18, 06002 (2011)

DOI: $10.1051 /$ epjconf/20111806002

(C) Owned by the authors, published by EDP Sciences, 2011

\title{
Open problems and perspectives offered by current and future instruments
}

\author{
M. Gérin ${ }^{\mathrm{a}}$
}

LERMA, CNRS UMR8112, Observatoire de Paris, École Normale Supérieure and Université Pierre et Marie Curie, 24 rue Lhomond, 75231 Paris Cedex 05, France

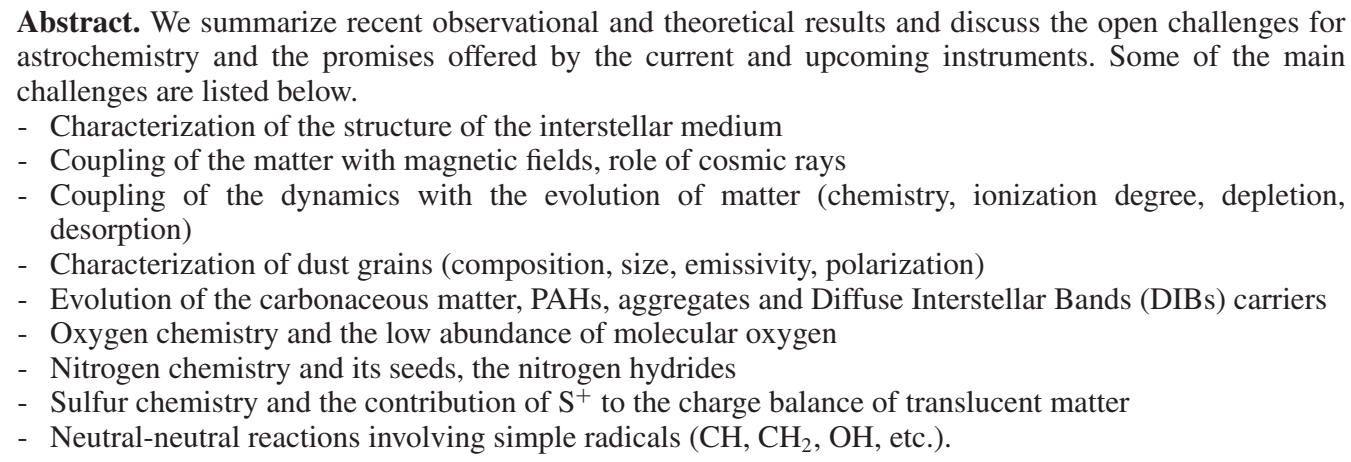

\section{ASTROCHEMISTRY AS A FIELD OF ASTROPHYSICS}

The future directions of research in astrophysics have been defined recently, both at the European (under the umbrella of the ASTRONET network ${ }^{1}$ ) and national levels ${ }^{2}$. Also the US astronomical decadal survey provides the main scientific directions for the future and recommendations on the development of instruments ${ }^{3}$. The main scientific themes have been summarized by the ASTRONET panels in four questions:

- Do we understand the extremes of the Universe ?

- How do galaxies form and evolve?

- What is the origin and evolution of stars and planets?

- How do we fit in ?

The research in Astrochemistry is mostly connected to the last two questions. Astrochemistry is a central topic for the research on the evolution of the matter, as well as the formation and evolution of stars and planets. It is connected, in a broader sense, to the evolution of galaxies, as star formation is one of the main drivers of galaxy evolution. The research in astrochemistry can be divided into two

\footnotetext{
ae-mail: maryvonne.gerin@ens.fr

1 see http://www. astronet-eu.org

2 led by CNRS/INSU http://www.insu.cnrs.fr

3 http://sites.nationalacademies.org/bpa

This is an Open Access article distributed under the terms of the Creative Commons Attribution-Noncommercial License 3.0, which permits unrestricted use, distribution, and reproduction in any noncommercial medium, provided the original work is properly cited.
} 


\section{EPJ Web of Conferences}

broad topics:

- Evolution of the matter:

This topic includes the evolution of the solid matter in all its forms (silicates, carbonaceous dust, volatile dust mantles), as well as the evolution of gas-phase species, and the relation between the solid and gas phases (formation and complexification of molecules, and processing by radiation and energetic particles, etc.)

- Dynamics of astrophysical diffuse media :

The chemical composition of diffuse media determines their energetic budget and controls their coupling to magnetic fields through the abundance of charged species. The coupling of the chemistry and dynamics of astrophysical media, from the largest scale of the objects themselves (galaxy, molecular cloud, proto-planetary disk) down to the very small scales where turbulence dissipates, has been identified as a key issue for future research. This topic includes the understanding of shocks, photon-dominated regions, disk dynamics, etc.

The numerous spectral features produced by the atoms, molecules and grains present in astrophysical objects are one of the main sources of information on these objects. In addition to determining the composition of matter (gas and/or solid), spectral lines provide information on the structure (column density, density, temperature, pressure), kinematics (through line profiles), magnetic fields (through polarization or Zeeman splitting), electron abundance, cosmic ray ionization rate $\zeta$.

As we will discuss in this paper, many of these diagnostics are well established, but their interpretation relies on the knowledge of both the formation process of the molecule involved and the line excitation mechanism. Both effects introduce uncertainties when one wants to derive physical or chemical parameters from the observed signals. The next step for the future is to better understand the sources of these uncertainties and reduce them in order to produce quantitative measurements that can be compared to state of the art models. This remark also applies to grains, since their composition and size distribution are also required to properly interprete the astrophysical signals.

\section{DETECTING MOLECULES}

The discovery of extraterrestrial molecules in the first half of the $20^{\text {th }}$ century was a surprise as these environments (first cometary comae, and then the interstellar medium) were perceived as very hostile for an active chemistry to operate. Further exploration of the variety of interstellar medium environments combined with the development of sensitive devices over a large fraction of the electromagnetic spectrum led to the current inventory of interstellar and circumstellar molecules (see Table 1 in the contribution by J.C. Guillemin). This inventory is far from being complete as several selection biases affect the possibility to identify and/or probe a significant fraction of molecular species. The majority of molecules have been identified with their centimeter/millimeter/submillimeter lines, that can be mostly assigned to rotational transitions. With state of the art sensitivity, these signals can only be detected for molecules with a permanent dipole moment. Molecular oxygen has no permanent electric dipole moment but its quadrupole magnetic moment is strong enough that rotational transitions are accessible to current instruments. Non polar molecules are therefore significantly more difficult to detect than polar species. In some instances, UV and visible spectroscopy of electronic transitions (e.g. $\mathrm{H}_{2}$ or $\mathrm{C}_{2}$ ), or IR spectroscopy of vibrational lines (e.g. $\mathrm{C}_{2} \mathrm{H}_{2}$ ) can be done, but the number of species accessible with these techniques is much more limited than the number of species accessible with radio techniques. This technical bias therefore introduces a bias in the "discovery space" of interstellar and circumstellar molecules. The bias is less pronounced for planetary atmospheres where the exploration of the infrared spectrum by ground based or space telescopes and by orbiting probes has led to a good characterization of their composition.

The detection of negative ions of long carbon chains is a very good example of a second bias, spectral confusion. In sources with a large number of molecules, the spectra get very crowded with a large number of spectral lines overlapping with each other. This line background is sometimes referred 
to as "weeds" that prohibit the detection of "flowers", the astronomically interesting molecules. In such sources, molecules with a relatively small number of intense lines (e.g. linear species with a large dipole moment) will be more easily identified than molecules with dense spectra, because (relatively) strong and isolated lines can be more reliably extracted from a line background than multiple weak lines. On the contrary, spectral signatures of molecules with a large number of spectral lines, as is often the case for organic species with internal rotors (e.g. $\left.\left(\mathrm{CH}_{3}\right)_{2} \mathrm{O}\right)$, become difficult to extract from a background of many lines. Isotopologues, defined as molecules bearing an isotopic substitute of at least one of their atoms, contribute for a large fraction to this background of low intensity spectral lines. Their identification and analysis are scientifically very interesting as isotopologues bear signatures of the formation processes of their parent molecules, and in addition can provide information on the isotopic ratio of different elements and/or on chemical processes leading to isotopic fractionation. Recent tentative detections of $\mathrm{DCOOCH}_{3}$ [12] and of the ${ }^{13} \mathrm{C}$ substitutes of methyl formate $\mathrm{HCOOCH}_{3}$ [7] illustrate the difficulty of detecting complex molecules in densely populated spectra.

\section{MOLECULES AND GRAINS AS PROBES OF ASTROPHYSICAL ENVIRONMENTS}

The richness of the spectra of astronomical objects gives many tools to understand their properties. Molecular spectral lines bear information on the medium where they are formed in addition to the information on the molecule itself (identification, column density). The combination of these information is one of the main tools used by astronomers to study interstellar and circumstellar environments. This applies to spectral lines of gas phase molecules, but is also relevant to some degree for solid-phase species as the spectral shapes of solid features also provide information on the temperature and composition of the solid material.

A combined analysis of the dust continuum emission and carefully selected spectral lines is the state of the art method for probing the density and temperature structure of various kinds of astrophysical sources [14]. Indeed, under the assumption that the gas and dust are well mixed, the analysis of the millimeter and submillimeter spectral energy distribution gives access to the dust temperature and column density, that can be inverted to provide density profiles. The molecular excitation is sensitive to the gas density and temperature as well. Therefore both diagnostics complete each other well.

These analysis are lengthy and require sophisticated models that take into account the geometrical structure of the studied objects. The current challenges are therefore twofold:

- on the astronomical side, the development of reliable source models that can be used on a wide basis.

- on the physics and chemistry side, the determination of accurate parameters for the dust optical properties, the molecular spectroscopic properties and collisional cross sections as a function of the temperature, for different collision partners $\left(\mathrm{He}, \mathrm{p}-\mathrm{H}_{2}, \mathrm{o}-\mathrm{H}_{2}\right)$.

\subsection{Structural properties}

The understanding of the structure of the interstellar medium is largely based on the statistical analysis of either (sub)millimeter images of dust continuum emission, or molecular line maps over sky areas that become increasingly larger with the improvement of detector sensitivities and the development of detector arrays. The ground state line of carbon monoxide, (CO $J=1 \rightarrow 0$ with a rest frequency $v=115.27 \mathrm{GHz}$, and a wavelength $\lambda=2.6 \mathrm{~mm}$ ), is widely used for tracing the structural properties of the velocity field $[21,26]$ while the sub-millimeter dust emission (or the visible/near IR dust extinction) reveals the distribution of the total column density of matter along the line of sight, with no information on the dynamics. These analyses have shown that the interstellar medium (ISM) is turbulent, and has remarkable scaling properties [43]. The characterization of the statistical properties of the ISM requires to cover a wide range of spatial scales from the smallest structures only accessible to millimeter interferometers up to the scale of molecular clouds themselves, over several decades in linear scales. The full benefit of these observations will only be obtained when the microscopic processes leading to the 

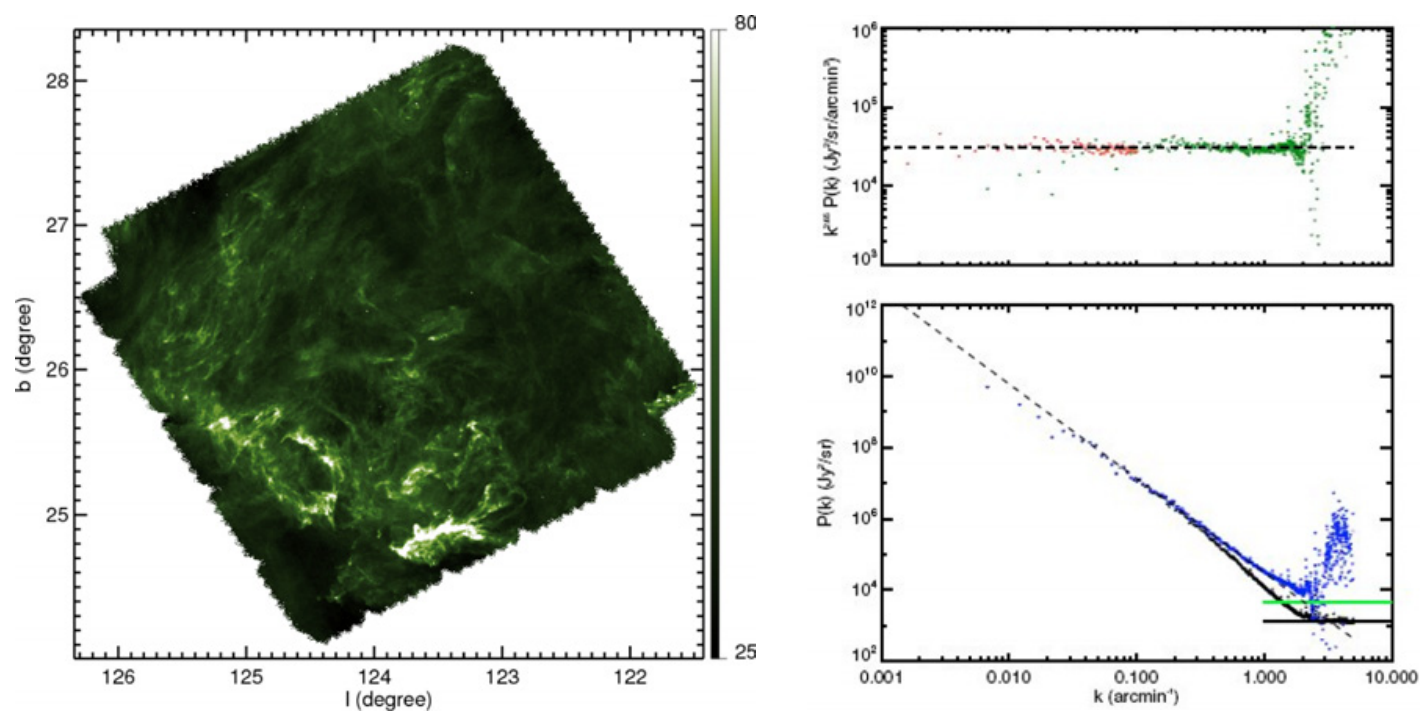

Figure 1. Left : Herschel submillimeter image of a diffuse cloud at high Galactic Latitude. Right : power spectrum of the combination of the Herschel and IRAS image. The same scaling law is found over three decades of spatial scales $[42,43]$.

formation of the detected signals will be completely elucidated. Regarding dust grains, observational evidences of variations of their emissivity properties with the grain temperature have been accumulated over the years (e.g. [46]). The detection of excess emission at centimeter to millimeter wavelengths [3] is another recent discovery that calls for a deeper understanding of interstellar grains, their composition, structural, thermal and electromagnetic properties. Both phenomena (emissivity and excess emission) show that state of the art models of interstellar dust grains such as the widely used DUSTEM code ${ }^{4}$ [9] are not fully valid and need to be upgraded with a better physical description of the optical properties of these nanograins. For instance, new models have been developed in which the variation of the dust emissivity with the grain temperature at submillimeter wavelengths is related to quantum effects in amorphous materials [41]. Also, a significant fraction of the $\mathrm{cm}$ excess emission is thought to be produced by spinning very small dust grains such as PAHs [13, 28, 54]. These models provide reasonable fits to available data, but the number of studied objects is too small for their full validation, and the number of parameters to fit in the models rather large due to our still partial knowledge of the nature of interstellar dust.

\subsection{Magnetic field}

Submillimeter images reveal that the interstellar matter is organized in a network of filaments whose orientation is correlated with the direction of the magnetic field [21, 42, 47]. Indeed, interstellar turbulence must be understood in the framework of the magnetohydrodynamics as the magnetic field is dynamically important. The study of the magnetic field direction and intensity, and its influence on the evolution of the matter and the star formation process is becoming an active research field. Information on the magnetic field properties are deduced from the polarization of the detected radiation, using dust emission or extinction, or the Zeeman effect on gas lines.

${ }^{4}$ http://www.ias.u-psud.fr/DUSTEM 

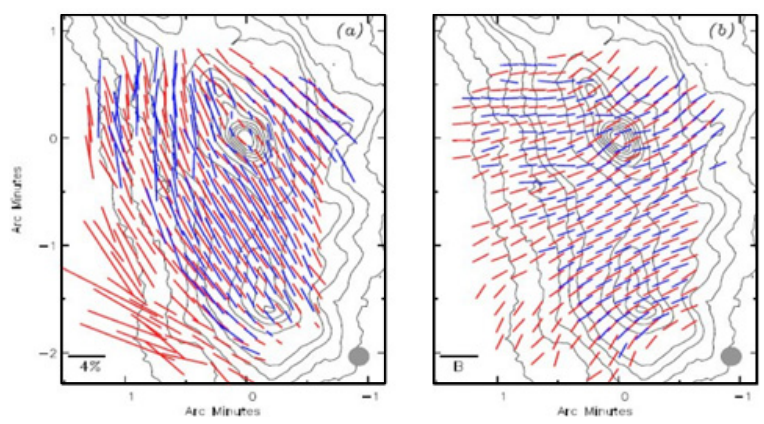

Figure 2. Maps of the dust polarization towards the OMC1 molecular cloud at two wavelengths : blue $350 \mu \mathrm{m}$, red $450 \mu \mathrm{m}$. The left image shows the dust polarization. Polarization vectors have been rotated by $90^{\circ}$ on the right image to show the orientation of the projected magnetic field [24].

Polarization of the dust emission or extinction results from the partial alignment of dust grains along the magnetic field. Polarization maps of star forming regions can be compared to results of numerical simulations. It is expected that, thanks to its full sky coverage and excellent sensitivity, the Planck satellite will revolutionize our understanding of the Galactic magnetic field, and its role in the condensation of insterstellar matter. The spatial resolution will be limited to $\approx 5^{\prime}$. On smaller spatial scales, dedicated instruments either using bolometer cameras on large single dish telescopes, or with sensitive radio interferometers (e.g. ALMA), need to be used. Recent advances in this field, prior to Planck launch, are summarized in [24]. The analysis of the maps of polarized dust continuum emission gives access to the direction of the magnetic field in the plane of the sky (the direction of polarization of the dust emission is perpendicular to the projected direction of the magnetic field). It is more difficult to extract an accurate information on the magnitude of the magnetic field however, because the mechanisms of grain alignment are still debated. The Chandrasekhar - Fermi method is using the statistics of the orientation of the magnetic field vectors, combined with the measurement of the velocity dispersion of the medium, to obtain an estimate of the magnitude of the magnetic field. It can be used to obtain the order of magnitude of the field strength but cannot be used for accurate measurements (see e.g. [29]).

The field intensity is best measured by measuring the modification of line profiles induced by the magnetic field. The Zeeman effect can now be detected using spectral lines of $\mathrm{HI}$ and $\mathrm{OH}$ in the centimeter domain, and $\mathrm{CN}$ lines in the millimeter region [10]. Although the sensitivity remains marginal, the current detections and upper limits enable to follow the increase of the field intensity with the volume density. The interpretation of the polarization data, especially the Zeeman splitting experiments, requires that the line formation mechanism is well understood. This calls for a dedicated effort to elucidate the chemistry of the magnetic field tracers : $\mathrm{HI}, \mathrm{OH}, \mathrm{CN}$. CN has the highest priority as the nitrogen chemistry is less well understood than either the carbon or oxygen chemistry, and especially the variation of the $\mathrm{CN}$ abundance with the gas density [27].

\subsection{Ionization fraction}

In the neutral interstellar gas, the ionization fraction, defined as the fractional abundance of charged species (either the negative charges that are mostly electrons, or the positively charge species that can be atomic or molecular ions) is thought to vary from $\sim 10^{-4}$ in the diffuse regions bathed by the FUV radiation field down to less than $\sim 10^{-8}$ in dense and well shielded cores. As the ionization fraction determines the ability of the matter to stay coupled with the magnetic field, it is very important to understand how it is regulated, and to be able to measure it in a broad range of environments to test those theoretical predictions. Measurements of the ionization fraction are always indirect and rely on chemical processes and reactions which efficiency depends on the ionization fraction. For instance, 

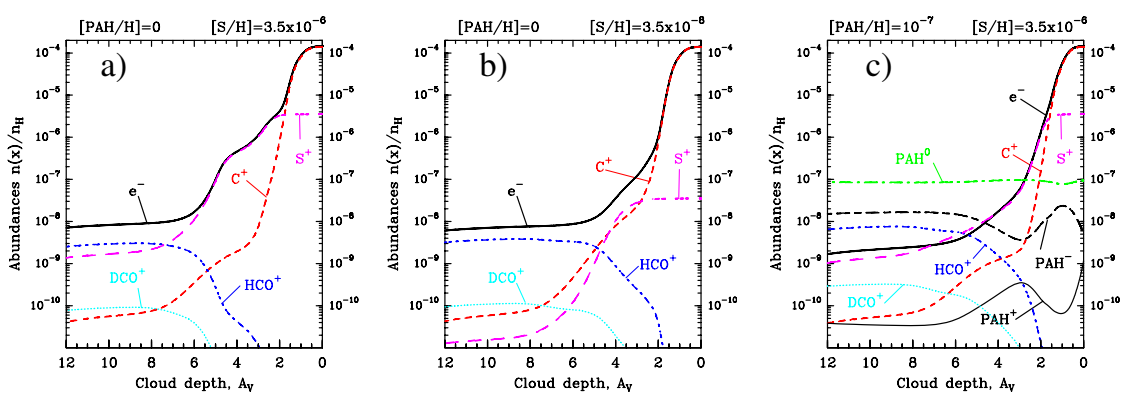

Figure 3. Variation of the ionization fraction as a function of depth into the molecular gas associated to the Horsehead nebula [20], for three cases. Left panel : standard model, no PAH and high sulfur abundance; Middle panel : no PAH, low sulfur abundance: Right panel : PAH and high sulfur abundance. The curves show the abundance of various charge carriers, $\mathrm{e}^{-}$full thick line, $\mathrm{C}^{+}$red dashed line, $\mathrm{S}^{+}$purple dashed line, $\mathrm{HCO}^{+}$blue dot-dashed line, $\mathrm{DCO}^{+}$cyan dotted line, $\mathrm{PAH}^{0}$ green line, $\mathrm{PAH}^{-}$black long dashed line, $\mathrm{PAH}^{+}$black line.

Goicoechea et al. [20] present observations of $\mathrm{HCO}^{+}, \mathrm{HOC}^{+}, \mathrm{H}^{13} \mathrm{CO}^{+}$and $\mathrm{DCO}^{+}$towards the Horsehead nebula and discuss how these molecular species provide diagnostics on the ionization fraction as a function of extinction in a molecular cloud. Indeed, abundances of molecular ions like $\mathrm{H}_{3}^{+}$, $\mathrm{HCO}^{+}$or $\mathrm{N}_{2} \mathrm{H}^{+}$are heavily dependent on the ionization fraction as these ions are rapidly destroyed by dissociative recombination reactions with electrons. Hence their abundances increase when the ionization fraction decreases. In dense and shielded regions, the hydrogen molecular ion $\mathrm{H}_{3}^{+}$and its deuterated substitutes $\left(\mathrm{H}_{2} \mathrm{D}^{+}, \mathrm{D}_{2} \mathrm{H}^{+}\right.$and $\left.\mathrm{D}_{3}^{+}\right)$are among the main carriers of positive charges. The deuterated hydrogen ions become very abundant in these cold regions thanks to efficient chemical enrichment reactions leading to an extremely efficient fractionation, and can transfer this enrichment to other ions and molecules, notably $\mathrm{HCO}^{+}\left(\mathrm{DCO}^{+}\right)$and $\mathrm{N}_{2} \mathrm{H}^{+}\left(\mathrm{N}_{2} \mathrm{D}^{+}\right)$. The deuterium fractionation is limited by both dissociative recombination reactions with electrons and by reactions of the deuterated ions with neutral species. This chemical pattern involves a limited number of reactions, and leads to an inverse relationship between the deuterium fractionation of molecular ions and the electron fraction [8, 23].

The diagnostic accuracy is limited by both the unknowns on the formation and destruction rates of molecular ions, and by the presence of other destruction paths than dissociative recombination, that limit the sensitivity of molecular abundances to the ionization fraction. Indeed, molecular ions can also be destroyed by reactions with abundant neutral species such as $\mathrm{CO}$. Furthermore, in the densest and coldest regions of dense cores, neutral species freeze onto grains forming their mantles. Hence the destruction rate of molecular ions by neutrals becomes lower and their abundance can be enhanced without any change in the electron fraction ([8]).

In UV-dominated regions, deuterium fractionation processes become less efficient because of the increased gas temperature, and because molecule destruction is dominated by FUV radiation. Other diagnostics must be found. Reactive ions like $\mathrm{CO}^{+}$or $\mathrm{HOC}^{+}$are good diagnostics of high electron abundances since these ions are destroyed by reactions with neutrals more rapidly than $\mathrm{HCO}^{+}$. They are therefore detected only in regions where ions are formed at a high rate. The abundance ratios $\mathrm{HOC}^{+} / \mathrm{HCO}^{+}$and $\mathrm{CO}^{+} / \mathrm{HCO}^{+}$steeply increase with increasing ionization fraction [20].

The use of chemical diagnostics however requires that the chemical processes be well understood in order to accurately calibrate such tools. This goal is far from being achieved : in dense cores, the dependence of reaction rates on temperature can lead to significant uncertainties. The largest source of error is however the role of grains, both as sinks for abundant atoms and molecules and as carriers of negative charges. As explained above, the depletion factors of abundant elements on grains significantly affect the chemistry, even when the ionization rate remains constant. PAHs and PAH aggregates are known to have a high electron affinity. Models have shown that, if they are present in dense cores, they may become the main carriers of negative charges rather than electrons. This has 
important consequences for the chemistry as negative PAHs interact differently than electrons with positive molecular ions [16]. Our understanding is not better for UV-illuminated regions : while the detection of reactive ions like $\mathrm{CO}^{+}, \mathrm{HOC}^{+}$is a clear sign of enhanced electron fraction, the formation of these species is not fully understood. Therefore their diagnostic power remains limited. Finally, because the ionization potential of sulfur is lower than that of carbon, ionized sulfur can be a major positive ion in moderately shielded regions, if sulfur does not deplete onto grains. This will affect the gradient in ionization fraction as a function of extinction. The sulfur chemistry remains a major issue in astrochemistry, because neither the gas phase, nor the solid phase budget of sulfur is fully elucidated. Therefore the depletion of sulfur from the gas phase remains largely unconstrained.

\subsection{Ionization rate and Cosmic rays}

Cosmic rays are thought to be the main source of ionization in molecular cores. They also contribute in initiating the chemistry in dense and diffuse regions by forming ionized $\mathrm{H}$ and $\mathrm{H}_{2}$ that can react with other species and build molecules through series of ion-molecule reactions. The interaction of cosmic rays (protons and electrons) with interstellar matter is highest for the lower energy particles, and can produce gamma rays [45]. Advances in high-energy instruments have been spectacular in the last decade with the development of the Fermi NASA space experiment, and the construction and operation of the HESS telescope in Namibia which is sensitive to very high energy gamma rays. Excellent promises lie ahead of us, with the upgrade of HESS to HESS2 and the design studies of the Cerenkov Telescope Array $\left(\mathrm{CTA}^{5}\right)$.

It is possible to obtain constraints on the cosmic ray ionization rate $\zeta$ by observing specific molecules that are chemically related to the products of cosmic ray ionization of atomic or molecular hydrogen. The list includes $\mathrm{H}_{3}^{+}$[31], as well as the recently detected molecular ions $\mathrm{OH}^{+}$and $\mathrm{H}_{2} \mathrm{O}^{+}[17,44]$. Extensive surveys of $\mathrm{H}_{3}^{+}$[31] have led to an upward revision of the cosmic ray ionization rate of $\zeta>10^{-16} \mathrm{~s}^{-1}$ in diffuse matter. Yet such a high rate is not compatible with the high deuterium fraction found in dense cores, where the currently adopted rate is $\zeta \sim 2 \times 10^{-17} \mathrm{~s}^{-1}[8,33]$. The natural conclusion is the existence of spatial variations of the cosmic ray density. Padovani et al. [45] present models of the propagation of cosmic rays in molecular gas. It is obvious that the constraints on the cosmic ray energy spectrum and propagation put by molecular line observations are not tight enough to select the best model and that more accurate molecular line observations and interpretation should help in understanding the cosmic ray origin and propagation.

\subsection{Probing chemical processes, isomers}

Modeling of the formation of interstellar molecules involves extensive chemical networks, in which a large fraction of reactions have poorly known rates or branching ratios. The applicability of reaction kinetics to very diluted systems such as the ISM is another important issue. As explained in J. Le Bourlot's contribution, most models compute either steady state or time dependent chemistry within a given (simplified) geometrical structure. Given the complexity of these networks, it is important to be able to probe specific processes, rather than addressing the full problem at once. One of the methods used by astronomers to provide constraints on the chemical paths is the measurements of abundance ratios of isotopologues such as $\mathrm{DCO}^{+}$and $\mathrm{HCO}^{+}$. The underlying reasoning is based on the hypothesis that the isotopologues are formed by very similar networks, and that the abundance ratios is governed by a limited number of fractionation reactions. This hypothesis is not perfectly valid because isotopic substitution can lead to different reaction pathways in exceptional case, but is to first order largely correct. The study of isotopologues involving isotopic substitutions of one of their heavy atoms

5 http://www.cta-observatory.org 

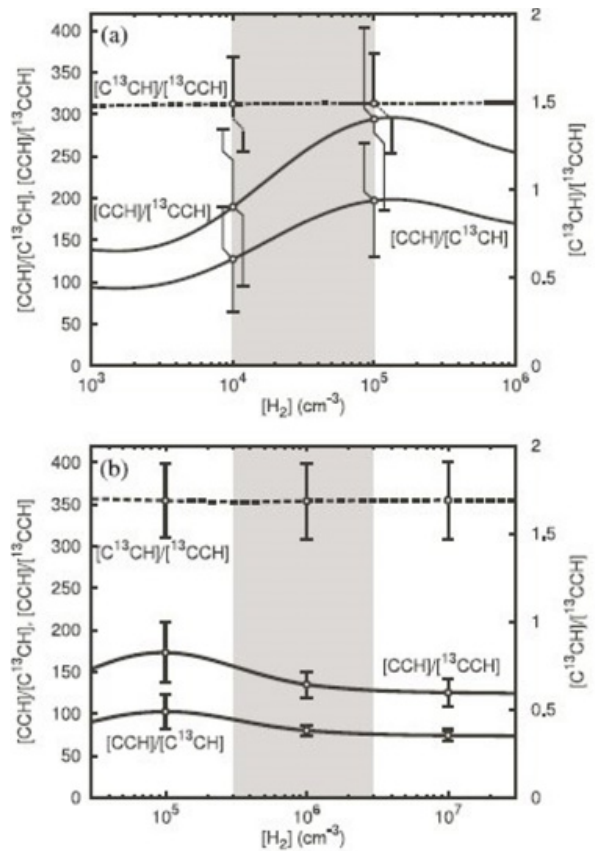

Figure 4. Comparison of the abundances of the $\mathrm{C}^{13} \mathrm{CH}$ and ${ }^{13} \mathrm{CCH}$ isomers in the dense cores TMC-1 and L1527. The excess of $\mathrm{C}^{13} \mathrm{CH}$ over ${ }^{13} \mathrm{CCH}$ indicates that the formation of $\mathrm{CCH}$ involves the $\mathrm{C}+\mathrm{CH}_{2}$ reaction where the two carbon atoms play different roles, together with other pathways where the carbon atoms are symmetrical [48].

$(\mathrm{C}, \mathrm{N}, \mathrm{O})$ can bring interesting constraints on the formation pathways of molecules. For instance, the detection of an excess in one of the ${ }^{13} \mathrm{C}$ derivatives of the ethynyl radical $\mathrm{CCH}, \mathrm{C}^{13} \mathrm{CH} /{ }^{13} \mathrm{CCH} \sim 1.6$ (Sakai et al. 2010) shows that a reaction where the two carbon atoms do not play equivalent role must play a significant role in the formation of interstellar $\mathrm{CCH}$. The authors suggest that the reaction $\mathrm{C}+\mathrm{CH}_{2} \rightarrow \mathrm{CCH}+\mathrm{H}$ is an important pathway for building $\mathrm{CCH}$. With the increased sensitivity of millimeter telescopes, such studies will certainly become more numerous in the future. Furthermore, the detection of several isomeric forms of molecules sharing the same raw chemical formula becomes now possible. The comparison of abundances of those isomers provides strong constraints on the chemical networks, as discussed by Marcelino et al. [40] for the case of HNCO and its isomers HOCN and HCON.

\section{OPEN PROBLEMS}

The strong couplings between the microscopical processes and the interstellar medium dynamics and thermal properties are one of the main difficulties in the understanding of its evolution. This includes the formation of stars and planets, as well as, at larger scales the evolution of galaxies because star formation is among the main driving forces of galaxy evolution. The work can be focused on open burning questions, that are all related to the topic of coupling the chemistry with the gas dynamics.

- Seeing the "dark" gas :

Classical tracers of the neutral interstellar gas include the hyperfine transition of atomic hydrogen at $21 \mathrm{~cm}$, and the carbon monoxide $(\mathrm{CO})$ rotational lines for molecular gas. The dust emission and absorption (extinction) is also used under the hypothesis that the gas and dust are well mixed. However, there is increasing evidence that $\mathrm{CO}$ is not a fully reliable tracer of molecular gas and that "dark" gas is present on the outskirts of CO clouds, "dark" being defined as molecular gas not radiating in $\mathrm{CO}$ [22]. Indeed, detailed comparisons of tracers of the total gas column through either 
the dust emission/absorption or the $\gamma$-ray maps, have revealed that the matter traced by $\mathrm{HI}$ and/or $\mathrm{CO}$ could not account for the totality of the detected signals, and therefore require the presence of molecular gas not traced by $\mathrm{CO}$. It is known from direct observations of $\mathrm{H}_{2}$ at far ultraviolet wavelengths that the onset of $\mathrm{CO}$ formation is not coincident with the onset of $\mathrm{H}_{2}$ formation. This behavior is well reproduced by chemical models [36, 53]. The detection of the "dark gas" is another confirmation of the difference between molecular gas and CO dominated gas. The FUV data are sparse and limited to relatively local sources. Herschel offers an alternative tracer of this matter, namely the fine structure transition of $\mathrm{C}^{+}$at $1.9 \mathrm{THz}(158 \mu \mathrm{m})$. Because $\mathrm{C}^{+}$is the main carbon reservoir in such regions, it can be used as a complementary tracer of molecular gas. In the framework of the GOTC+ key program, the Herschel satellite is currently providing excellent $\mathrm{C}^{+}$data, fully revealing the presence and dynamics of this new interstellar gas component [35].

- Formation of molecules in diffuse matter:

The diffuse ISM is highly turbulent and bathed by FUV radiation. It is therefore remarkable that molecules can be formed in such an hostile environment and survive with detectable abundances. While the framework of steady state chemistry accounts rather well for the formation of molecules, it does not provide a fully consistent picture. The intensity of the ground state rotational line of CO presents large fluctuations in low extinction gas, that are unrelated to the variation of the total column density of matter but rather trace the regions where the formation of $\mathrm{CO}$ is enhanced. The behavior of CO illustrates the need for coupling the gas chemistry with its dynamics $[15,38]$. The carbon, nitrogen and oxygen hydrides are formed in the first steps of chemical networks and are as such at the roots of interstellar chemistry. Thanks to its broad wavelength coverage, the Herschel satellite offers very powerful instruments for probing hydrides. First results, published in the Astronomy and Astrophysics Herschel special issue ${ }^{6}$, confirm the presence of previously detected species $(\mathrm{CH}$, $\left.\mathrm{CH}^{+}, \mathrm{OH}, \mathrm{H}_{2} \mathrm{O}, \mathrm{NH}, \mathrm{NH}_{2}, \mathrm{NH}_{3}, \mathrm{HF}\right)$ and report new species $\left(\mathrm{OH}^{+}\right.$also detected simultaneously from the ground, $\mathrm{H}_{2} \mathrm{O}^{+}, \mathrm{H}_{2} \mathrm{Cl}^{+}$). The overabundance of $\mathrm{OH}^{+}$relative to $\mathrm{H}_{2} \mathrm{O}^{+}$indicates that $\mathrm{OH}^{+}$ and $\mathrm{H}_{2} \mathrm{O}^{+}$originate from regions where the fraction of molecular hydrogen is very small (less than $10 \%$ ). Together with $\mathrm{CH}, \mathrm{HF}$ is scaling linearly with the column density of molecular hydrogen. Both molecules can therefore be used as tracer of molecular gas, more reliably than $\mathrm{CO}$. With its large dipole moment, $\mathrm{HF}$ is more sensitive than $\mathrm{CH}$, reaching an opacity of order unity for an $\mathrm{H}_{2}$ column density as low as $10^{20} \mathrm{~cm}^{-2}$ and a velocity dispersion of $1 \mathrm{~km} \mathrm{~s}^{-1}$. The Herschel $\mathrm{CH}^{+}$observations confirmed the trends obtained from visible spectroscopy, namely the ubiquity of this reactive ion in the diffuse ISM.

New mysteries have arisen, for instance the very similar abundances of the $\mathrm{N}$-hydrides $\mathrm{NH}, \mathrm{NH}_{2}$ and $\mathrm{NH}_{3}$, or the tight correlation of $\mathrm{CH}, \mathrm{CCH}$ and $\mathrm{c}-\mathrm{C}_{3} \mathrm{H}_{2}$. Models aiming at predicting the observed distribution and abundances of hydrides self consistently must therefore become more realistic. Models including the specific chemistry taking place in the dissipative regions of interstellar turbulence lead to better predictions for many molecules, most notably $\mathrm{CH}^{+}$, than standard PDR models [19]. Indeed, although such regions occupy a very small fraction of the total volume, they host specific conditions for a short time, when new chemical pathways for the molecule formation are opened. Despite their low filling factors, they can therefore dominate the formation of some species, and have a significant impact on the abundances of a large fraction of the molecules detected in the diffuse ISM.

- Molecular oxygen:

The oxygen budget in the interstellar medium is discussed in [32, 52]. When accounting for the main reservoirs in the solid phase (silicates, water ice, $\mathrm{CO}, \mathrm{CO}_{2}$ and $\mathrm{CH}_{3} \mathrm{OH}$ ice) and in the gas $(\mathrm{CO}$, atomic oxygen, $\mathrm{H}_{2} \mathrm{O}$ ), a missing oxygen reservoir remains. This cannot be molecular oxygen since the very deep observations performed by SWAS, ODIN and now Herschel lead to upper limits of the

6 Volumes 518 and 521. 


\section{EPJ Web of Conferences}

molecular oxygen abundance of $\approx 10^{-7}$ or even lower. This low abundance can be explained if oxygen gets quickly depleted from the gas phase to build the ice mantles as in the models presented by [25]. However, these models still predict that molecular oxygen should exist in an intermediate layer where the extinction is large enough to shield this molecule from FUV radiation and the density not too high so that the depletion on dust grains is not efficient. Deeper observations performed with Herschel will be able to probe these models. If molecular oxygen remains undetected, new explanations will be needed. Once again, coupling of the gas dynamics with its chemistry may lead to important changes of the abundances of oxygen bearing molecules if the gas dynamics triggers the mixing of illuminated material rich in $\mathrm{C}$ and $\mathrm{C}^{+}$with shielded gas rich in $\mathrm{CO}$ and $\mathrm{O}_{2}$. Numerical models simulating the formation of molecular clouds can now be computed with limited chemical networks [18]. Although the method is still limited by computing power, it may be very interesting to test how the heterogenous structure of interstellar clouds affects the oxygen chemistry using such codes.

- Growth of molecular complexity:

The formation of "complex", mostly organic, molecules is a key question for understanding the interplay between the gas and solid phases in the dense interstellar matter. In this astrophysical context, "complex" means organic molecules with three or more heavy atoms, having a chemical function (e.g. alcohol or ketone) such as ethyl alcohol $\mathrm{CH}_{3} \mathrm{CH}_{2} \mathrm{OH}$, or amino-acetonitrile $\mathrm{NH}_{2} \mathrm{CHCN}$ [1]. As explained above, the identification of such molecules in spectra of astrophysical objects is hindered by spectral confusion. Although many relatively complex molecules have been detected, the inventory is probably not complete because the detection limit in terms of relative abundance depends on the characteristics of the emitted spectra. As sources where the complex organic molecules are detected are very compact, radio interferometers achieve lower spectral confusion than single dish telescopes because: $i$ ) the extended emission from abundant species is filtered out by the long interferometric base-lines, and $i$ ) different sources with specific chemistries are spatially separated $[4,23]$. ALMA is expected to give access to unprecedented spatial resolution (0.01") and a sensitivity ten times better than currently possible. The combination of both effects is expected to lead to lower confusion and identification of a larger number of new molecules. The far-infrared spectral range is a second promising spectral window for detecting new species, by accessing low lying torsional or vibrational modes (near $100 \mathrm{~cm}^{-1}$ ). These modes are particularly interesting for species not easily detected in the millimeter or submillimeter domains.

- Carriers of the Diffuse Interstellar Bands:

The diffuse interstellar bands (DIBs) are unidentified spectral features, detected in the visible spectra of bright stars, that are broader than interstellar atomic or molecular lines, but too narrow to be stellar lines. It is widely believed that their carriers are made of carbon, either as molecules or larger systems such as PAHs aggregates or fullerenes because these systems can resist the interstellar radiation field. The search of individual species is difficult because the neutral form is usually not the most stable one in interstellar conditions, and ionized or deshydrogenated species can be preferred. Thanks to the improvement in the accuracy and sensitivity of spectrometers, very weak lines can now be detected. The tentative identification of the $\mathrm{HC}_{4} \mathrm{H}^{+}$cation has been recently reported [34]. Iglesias-Groth et al. [30] also report tentative detections of two small PAH cations towards a specific line of sight with anomalous excess centimeter emission. Finally, the analysis of Spitzer IRS spectra has led to the identification of IR emission bands of fullerenes $\left(\mathrm{C}_{60}\right.$ and $\left.\mathrm{C}_{70}\right)$ in reflection nebulae [50] and a proto-planetary nebula [6]. These recent progresses show that the probability of having confirmed identification of some DIBs is getting higher, after decades of intense research. Dedicated spectroscopic work is needed for confirming the tentative identifications, that now reaches the required sensitivity [39].

- Evolution of the carbonaceous solid matter:

In the ISM, the largest carbon reservoir is the solid carbonaceous matter that accounts for approximatively two-third of the elemental carbon abundance $\left(\mathrm{C} / \mathrm{H}=3 \times 10^{-4}\right)$, while gas phase carbon in all its forms $\left(\mathrm{C}^{+}, \mathrm{C}, \mathrm{CO}\right.$, etc.) accounts for one third. Therefore, the study of the evolution of 
the solid carbonaceous matter and its interplay with the gas phase is a key question. It is closely related to the identification of DIB carriers, as the most likely carriers are large carbonaceous molecules, but it has wider implications. The analysis of the spectral features attributed to carbonaceous matter across the electromagnetic spectrum, notably the $2175 \AA$ UV bump, the $3.4 \mu \mathrm{m}$ absorption feature, the Aromatic Infrared Bands (AIBs) at 3.3, 6.2 7.7, 8.6, $11.3 \mu \mathrm{m} .$. , show that the shape and intensity of spectral features change with the environment where they are detected. These modifications have been attributed to evolution of the carbonaceous matter under the processing of FUV radiation, cosmic rays, shocks etc. The solar system organic matter, probed in comets, meteorites or asteroids, also presents indications of active processing. By combining laboratory experiments with detailed astronomical studies, a general understanding of the processes controlling the evolution of carbonaceous matter has been gradually built. These advances are reported in other contributions in this book, especially those of K. Demyk and L. Remusat. The carbonaceous matter is found to be composed of amorphous hydrogenated carbons (HAC, [11]) and polycyclic aromatic hydrocarbons (PAHs [51]) either isolated or in clusters. Fullerenes [6,50] complete the inventory. Detailed analyses of the evolution of the spectral features in photodissociation region show a clear spatial evolution connected to the intensity of the radiation field, from ionized PAHs in FUV illuminated regions, to neutral species, and then to $\mathrm{PAH}$-containing nanograins in the slightly shielded region, close to the $\mathrm{H} / \mathrm{H}_{2}$ interface [2].

\section{PERSPECTIVES WITH CURRENT AND FUTURE INSTRUMENTS, AND SUMMARY}

The open questions listed above can be put in close correspondence with state of the art instruments or upcoming projects. Instruments operating at infrared, submillimeter and millimeter wavelengths are particularly interesting for the field of astrochemistry. The Herschel space telescope ${ }^{7}$ is gathering an impressive harvest of scientific results, addressing the gas and solid phase composition, as well as the structure and dynamics of the objects. The Planck cosmological satellite ${ }^{8}$, launched simultaneously with Herschel, is providing unprecedented data on the dust emission in the Milky Way, as a foreground of the Cosmic Background signal. After the success of the ISO and Spitzer infrared missions, the James Webb Space Telescope (JWST) ${ }^{9}$ is expected to lead to new progresses with its very large surface and sensitivity. If its selection is confirmed, the Japanese led project SPICA, a cooled $3 \mathrm{~m}$ telescope ${ }^{10}$, would provide a tenfold increase in sensitivity compared to Herschel for photometry and medium/low resolution spectroscopy.

Tremendous progresses are also expected with ground based telescopes. The Atacama Large Millimeter/submillimeter Array (ALMA) ${ }^{11}$ is starting to operate with a restricted number of antennas, until it reaches full capacity near 2014. With its broad frequency coverage (atmospheric windows from $\sim 80 \mathrm{GHz}$ to $\sim 900 \mathrm{GHz}$, or even down to $30 \mathrm{GHz}$ ), it will offer unprecedented capabilities for spectroscopy and high spatial resolution imaging. A significant upgrade of the IRAM plateau de Bure Interferometer, i.e. a doubling of the number of telescopes, has been recommended in the French future planning.

It is certain that scientific discoveries resulting from these new projects will be so numerous, that the demand for more advanced modeling tools, and for more extensive databases (spectroscopy, reactivity, etc.) will steeply increase. In particular, the increased accuracy of the measurements will call for a matching increase in the accuracy of the modeling and of the comparison tools between models/simulations and observations. Efforts need to be made at all steps in the construction of

\footnotetext{
${ }^{7}$ http://herschel.esac.esa.int/

8 www.rssd.esa.int/Planck/

${ }^{9}$ http://www.jwst.nasa.gov

10 http://www.ir.isas.jaxa.jp/SPICA/SPICA_HP/index_English.html

11 http://www.almaobservatory.org
} 

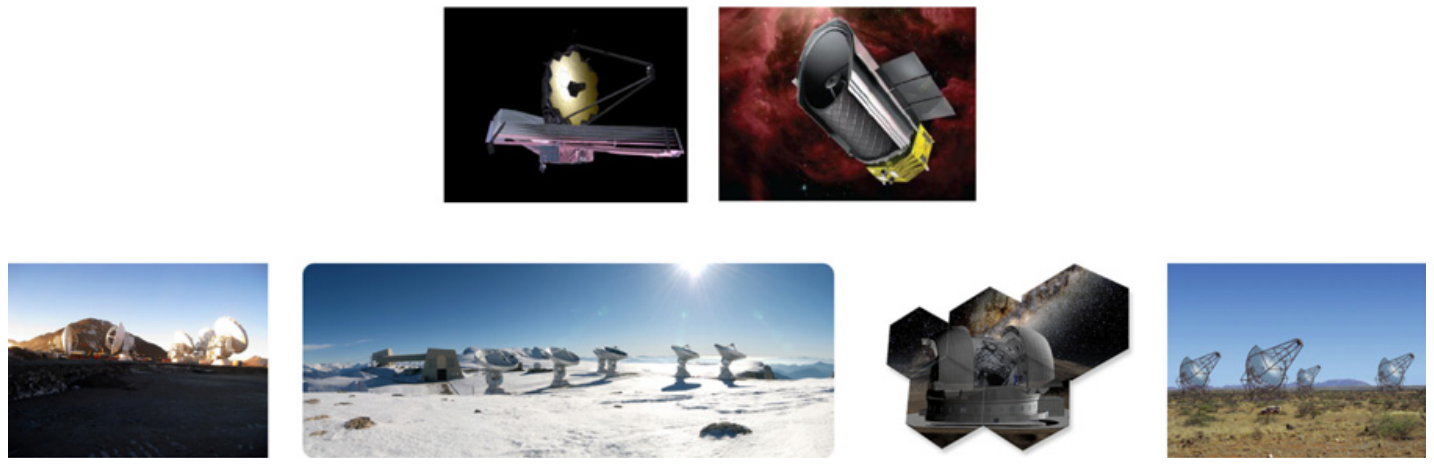

Figure 5. Sketches of planned or proposed future telescopes. From left to right, top row : the space telescopes JWST and SPICA; bottom row : the ground based telescopes ALMA (in construction), Extremely Large Telescope (ELT, project), Plateau de Bure Interferometer (PdBI) (in operation, proposal for extension as NOEMA), HESS as example for the CTA project.

the next generation of models: the understanding of the microscopic processes and their numerical implementation in models, the description of the environment of astrophysical objects and their evolution, including all relevant couplings (magnetic fields, radiations, cosmic rays, etc.), the calculation of the emerging signals that can be detected by telescopes over the electromagnetic spectrum (radiative transfer), and finally the modeling of the observation process and the comparison of models with observations.

This paper was prepared for the workshop "AstrOHP 2010, Chimie DANS Les Milieux astrophysiQues" held at Observatoire de Haute Provence in September 2010. We thank the organizers for the opened and friendly atmosphere during the workshop, and the numerous scientific discussions that took place. We also thank INSU/CNRS for the long support to the PCMI "Physique et Chimie du Milieu Interstellaire" programme that contributed to the development of the scientific exchanges and collaborations between astrophysics, physics and chemistry over the last twenty years.

\section{References}

[1] Belloche A., Menten K. et al. A\&A 482, (2008), 179

[2] Berné O. et al. A\&A (2007)

[3] Bot C., Ysard N., et al., A\&A 523,(2010) 20.

[4] Braakman A., Belloche A. et al. ApJ 724 (2010) 994.

[5] Burgh E.B., France K., Mc Candiss R.R., ApJ 658 (2007) 446

[6] Cami J. et al. Science, 329 (2010) 1180

[7] Carjaval M. et al. , A\&A 500, (2009), 1109

[8] Caselli P. et al., A\&A 492 (2008), 703

[9] Compiègne M., Verstraete L., Bernard J. Ph., et al., A\&A in press (2010), arxiv 1010.2769

[10] Crutcher R.M. Rev Mex AA 36, (2009), 107

[11] Dartois E., Muñoz-Caro G/M., A\&A 476 (2007) 1235.

[12] Demyk K. et al., A\&A 517, (2010), 17

[13] Draine B., Lazarian A., 1998, ApJ 508, 157.

[14] van Dishoeck E., Herbst E. ARAA 47, (2010), 427. 
[15] Falgarone E., Pety J., Hily-Blant P. . A\&A 507 (2009) 355.

[16] Flower D.R., Pineau des Forêts G., Walmsley C.M., A\&A 474, (2007), 923.

[17] Gerin M. et al., 2010 A\&A 518 L110.

[18] Glover S. C.O. et al. MNRAS 404 (2010) 2

[19] Godard B., Falgarone E., Pineau des Forêts G. A\& 495 (2009) 847

[20] Goicoechea J.R., Pety J., Gerin M., et al. A\&A 498, (2009), 771

[21] Goldsmith P., Heyer M., et al. ApJ 680 (2008) 428

[22] Grenier I., Casandjian, J.-M., Terrier, R., Science 307 (2005) 1292.

[23] Guélin M., Langer W.D., Wilson R.W., A\&A 107, (1982), 107.

[24] Hildebrand R. \& Vaillancourt J.E. Rev Mex AA 36, (2009, 137

[25] Hollenbach D. et al. ApJ 690 (2009)1497

[26] Hily-Blant P., Falgarone E., Pety J. A\&A 481, (2008), 367.

[27] Hily-Blant P., Walmsley C.M., Pineau des Forêts G., Flower D., A\&A 513 (2010) 41

[28] Hoang T., Draine B.T., Lazarian A., ApJ 715, (2010), 1462

[29] Houde M., Vaillancourt J., Hildebrandt R., et al., ApJ 706, (2009) 1504.

[30] Iglesias-Groth et al. MNRAS 407 (2010) 2157.

[31] Indriolo N., Geballe T., Oka T. et al. ApJ 671 (2007), 1736.

[32] Jenkins E.B., ApJ 700 (2010), 1299.

[33] Keto E., Caselli P., MNRAS 402 (2010), 1625.

[34] Krełowski J. et al. ApJ 714 (2010) L64

[35] Langer W.D., et al. A\&A 521, (2010), L17

[36] Lequeux J. et al. A\&A 292 (1994), 371.

[37] Liszt H.S. A\&A 461 (2007) 205

[38] Liszt H.S., Pety J., Lucas R. A\&A 518 (2010) A 45

[39] Maier J.P., Walker G.A.H., Bohlender D.A. , arXiv 1011.0401

[40] Marcelino N., Brünken S., Cernicharo J. A\&A 516 (2010) A105

[41] Mény C., Gromov V., Boudet N. et al. A\&A 468 (2007) 171.

[42] Me'nshchikov A. et al. A\&A 518, (2010), 1103

[43] Miville-Deschênes M.A. et al., A\&A 518, (2010), L104

[44] Neufeld et al. A\&A 518 (2010) L108

[45] Padovani M., Galli D., Glassgold A.E. A\&A 501 (2009) 619

[46] Paradis D., Bernard J.Ph., Mény C., A\&A 506, (2009), 745

[47] Peyrera A., Magalhaes A.M., 2007, ApJ 662, 1014

[48] Sakai N., Saruwatari O., Sakai T, A\&A 512 (2010) A31

[49] Sheffer Y., Rogers M., Federman S. et al. ApJ 787 (2008) 1075

[50] Sellgren K. et al. ApJ 722 (2010), L54

[51] Tielens A.G.G.M., ARAA 46 (2008), 289.

[52] Whitett D.C.B., Apj 710 (2010) 1009

[53] Wolfire M G., Hollenbach D., Mc Kee C.F., ApJ 716 (2010), 1191

[54] Ysard N., Verstraete L., A\&A 509, (2010) A12. 